\title{
CHARACTERIZATION OF LIPSCHITZ-TYPE FUNCTIONS BY GARSIA-TYPE NORMS ON THE UPPER HALF SPACE
}

\author{
Jisoo Byun, Hong Rae Cho, And Bong-Hak Im
}

\begin{abstract}
It is well-known that the $B M O$ norm is equivalent to the Garsia norm. In this paper, we characterize mean-Lipschitz spaces by using Garsia-type norms on the upper half space $\mathbb{R}_{+}^{n+1}$.
\end{abstract}

\section{Introduction and statement of results}

Let $\mathbb{R}_{+}^{n+1}$ be the $(n+1)$-dimensional upper-half space. In the coordinate notation, we have

$$
\mathbb{R}_{+}^{n+1}=\left\{(x, t) \in \mathbb{R}^{n} \times \mathbb{R}: t>0\right\} .
$$

We can consider $\mathbb{R}^{n}$ as the boundary of $\mathbb{R}_{+}^{n+1}$. For $t>0$, we denote the Euclidean ball in $\mathbb{R}^{n}$ by

$$
Q_{t}(x)=\left\{y \in \mathbb{R}^{n}:|x-y|<t\right\}, \quad x \in \mathbb{R}^{n} .
$$

We define the integral mean $f_{Q_{t}}$ by

$$
f_{Q_{t}(x)}=\frac{1}{\left|Q_{t}(x)\right|} \int_{Q_{t}(x)} f(y) d y
$$

and the $B M O$ norm as

$$
\|f\|_{B M O}=\sup _{\substack{x \in \mathbb{R}^{n} \\ t>0}} \frac{1}{\left|Q_{t}(x)\right|} \int_{Q_{t}(x)}\left|f-f_{Q_{t}(x)}\right| d y .
$$

Here $\left|Q_{t}(x)\right|$ is the volume of $Q_{t}(x)$ in $\mathbb{R}^{n}$. The space $B M O$ of bounded mean oscillation is a set of all $L_{l o c}^{1}$ functions on $\mathbb{R}^{n}$ with the finite norm $\|f\|_{B M O}<\infty$.

The Poisson kernel in $\mathbb{R}_{+}^{n+1}$ has an explicit expression;

$$
P_{t}(x)=\frac{c_{n} t}{\left(|x|^{2}+t^{2}\right)^{(n+1) / 2}}, \quad c_{n}=\frac{\Gamma((n+1) / 2)}{\pi^{(n+1) / 2}} .
$$

Received October 6, 2011; Accepted January 11, 2012.

2000 Mathematics Subject Classification. 42B35.

Key words and phrases. upper half space, $B M O$, mean-Lipschitz spaces, Garsia-type norm.

This work was supported by a 2-Year Research Grant of Pusan National University. 
The Poisson integral of a function given on $\mathbb{R}^{n}$ is defined by

$$
\mathcal{P} f(x, t)=P_{t} * f(x)=\int_{y \in \mathbb{R}^{n}} P_{t}(x-y) f(y) d y .
$$

Garsia has observed that there is another norm for functions in $B M O$ which is easier to use.[3] For $f \in L_{\text {loc }}^{1}\left(\mathbb{R}^{n}\right)$, the Garsia norm $\mathcal{G}(f)$ is defined by

$$
\mathcal{G}(f)=\sup _{(x, t) \in \mathbb{R}_{+}^{n+1}} \int_{y \in \mathbb{R}^{n}}|f(y)-\mathcal{P} f(x, t)| P_{t}(x-y) d y .
$$

It is well-known that $[2]$

$$
\|f\|_{B M O} \sim \mathcal{G}(f) .
$$

For the unit ball in $\mathbb{C}^{n}$ the $B M O$ norm is defined by using the non-isotropic ball on the boundary of the unit ball. The same result as (1) on the unit ball in $\mathbb{C}^{n}$ was proved by Garsia (see [2], one-dimensional case) and by Axler-Shapiro (see [1], $n$-dimensional case).

Let $1 \leq p, q \leq \infty$ and $0<\alpha<1$. For $f \in L^{p}\left(\mathbb{R}^{n}\right)$, we denote

$$
\Delta_{\alpha}^{p, q}(f)=\left(\int_{y \in \mathbb{R}^{n}} \frac{\|f(\cdot+y)-f(\cdot)\|_{L^{p}\left(\mathbb{R}^{n}\right)}^{q}}{|y|^{n+\alpha q}} d y\right)^{1 / q}
$$

and we define the mean-Lipschitz norm by

$$
\|f\|_{\Lambda_{\alpha}^{p, q}}=\|f\|_{L^{p}\left(\mathbb{R}^{n}\right)}+\Delta_{\alpha}^{p, q}(f) .
$$

Then $\Lambda_{\alpha}^{p, q}$ consists of all functions $f$ in $L^{p}\left(\mathbb{R}^{n}\right)$ for which the norm $\|f\|_{\Lambda_{\alpha}^{p, q}}$ is finite. It is called the mean-Lipschitz space. For a measurable function $F$ on $\mathbb{R}_{+}^{n+1}$ we define

$$
L_{\alpha}^{p, q}(F)=\left(\int_{0}^{\infty}\left(t^{1-\alpha}\|F(\cdot, t)\|_{L^{p}\left(\mathbb{R}^{n}\right)}\right)^{q} \frac{d t}{t}\right)^{1 / q} .
$$

We note that [4]

$$
\int_{0}^{\infty}\left(t^{1-\alpha}\|\nabla \mathcal{P} f(\cdot, t)\|_{L^{p}\left(\mathbb{R}^{n}\right)}\right)^{q} \frac{d t}{t} \sim \int_{0}^{\infty}\left(t^{1-\alpha}\left\|\frac{\partial}{\partial t} \mathcal{P} f(\cdot, t)\right\|_{L^{p}\left(\mathbb{R}^{n}\right)}\right)^{q} \frac{d t}{t} .
$$

By Hardy-Littlewood lemma [4], we get

$$
\Delta_{\alpha}^{p, q}(f) \sim L_{\alpha}^{p, q}(\nabla \mathcal{P} f)
$$

Now, we define the Garsia-type $(p, q)$-norm by

$$
\begin{aligned}
& \mathcal{G}_{\alpha}^{p, q}(f) \\
= & \left(\int_{0}^{\infty} \frac{1}{t^{1+\alpha q}}\left(\int_{x \in \mathbb{R}^{n}}\left(\int_{y \in \mathbb{R}^{n}}|f(y)-\mathcal{P} f(x, t)| P_{t}(x-y) d y\right)^{p} d x\right)^{q / p} d t\right)^{1 / q} .
\end{aligned}
$$


When $q=\infty$, the expressions (2), (3), and (4) are interpreted in the normal limiting way, namely

$$
\begin{gathered}
\Delta_{\alpha}^{p, \infty}(f)=\sup _{|y|>0} \frac{\|f(\cdot+y)-f(\cdot)\|_{L^{p}\left(\mathbb{R}^{n}\right)}}{|y|^{\alpha}}, \\
L_{\alpha}^{p, \infty}(\nabla \mathcal{P} f)=\sup _{t>0} t^{1-\alpha}\|\nabla \mathcal{P} f(\cdot, t)\|_{L^{p}\left(\mathbb{R}^{n}\right)},
\end{gathered}
$$

and

$$
\mathcal{G}_{\alpha}^{p, \infty}(f)=\sup _{t>0} \frac{1}{t^{\alpha}}\left(\int_{x \in \mathbb{R}^{n}}\left(\int_{y \in \mathbb{R}^{n}}|f(y)-\mathcal{P} f(x, t)| P_{t}(x-y) d y\right)^{p} d x\right)^{1 / p} .
$$

Theorem 1.1. Let $1 \leq p, q \leq \infty$ and $0<\alpha<1$. For $f \in L^{p}\left(\mathbb{R}^{n}\right)$ we have

$$
\Delta_{\alpha}^{p, q}(f) \sim \mathcal{G}_{\alpha}^{p, q}(f) .
$$

Recall that the Poisson kernel for the upper half space is given by

$$
P_{t}(x-y)=\frac{c_{n} t}{\left(|x-y|^{2}+t^{2}\right)^{(n+1) / 2}} .
$$

Lemma 1.2. ([4]) Let $0<\alpha<1$. Then

(i) $\int_{\mathbb{R}^{n}}|x-y|^{\alpha} P_{t}(x-y) d y \lesssim t^{\alpha}$;

(ii) $\left|\nabla P_{t}(x-y)\right| \lesssim t^{-1} P_{t}(x-y)$ for all $(x, t) \in \mathbb{R}_{+}^{n+1}$ and for all $y \in \mathbb{R}^{n}$.

Lemma 1.3. (Hardy's inequalities) Let $h$ is a non-negative function and $p \geq 1, r>0$. Then we have

$$
\begin{aligned}
& \text { (i) }\left[\int_{0}^{\infty}\left(\int_{0}^{x} h(y) d y\right)^{p} x^{-r-1} d x\right]^{1 / p} \leq \frac{p}{r}\left(\int_{0}^{\infty}(y h(y))^{p} y^{-r-1} d y\right)^{1 / p} ; \\
& \text { (ii) }\left[\int_{0}^{\infty}\left(\int_{x}^{\infty} h(y) d y\right)^{p} x^{r-1} d x\right]^{1 / p} \leq \frac{p}{r}\left(\int_{0}^{\infty}(y h(y))^{p} y^{r-1} d y\right)^{1 / p} .
\end{aligned}
$$

\section{Proof of Theorem 1.1}

First we consider the case $p=q=\infty$.

For $(x, t) \in \mathbb{R}_{+}^{n+1}$ and $y \in \mathbb{R}^{n}$ we have

$$
|f(y)-\mathcal{P} f(x, t)| \lesssim|f(y)-f(x)|+|f(x)-\mathcal{P} f(x, t)|
$$

and

$$
\begin{aligned}
|f(x)-\mathcal{P} f(x, t)| & =\left|\int_{y \in \mathbb{R}^{n}}(f(x)-f(y)) P_{t}(x-y) d y\right| \\
& \lesssim \Delta_{\alpha}^{\infty, \infty}(f) \int_{y \in \mathbb{R}^{n}}|x-y|^{\alpha} P_{t}(x-y) d y \\
& \lesssim \Delta_{\alpha}^{\infty, \infty}(f) t^{\alpha},
\end{aligned}
$$


by (i) of Lemma 1.2. Thus we have

$$
|f(y)-\mathcal{P} f(x, t)| \lesssim \Delta_{\alpha}^{\infty, \infty}(f)\left(|x-y|^{\alpha}+t^{\alpha}\right) .
$$

By (i) of Lemma 1.2 again, we have

$$
\begin{aligned}
& \int_{y \in \mathbb{R}^{n}}|f(y)-\mathcal{P} f(x, t)| P_{t}(x-y) d y \\
& \quad \lesssim \Delta_{\alpha}^{\infty, \infty}(f)\left(\int_{y \in \mathbb{R}^{n}}|x-y|^{\alpha} P_{t}(x-y) d y+t^{\alpha}\right) \\
& \quad \lesssim \Delta_{\alpha}^{\infty, \infty}(f) t^{\alpha} .
\end{aligned}
$$

This implies that

$$
\mathcal{G}_{\alpha}^{\infty, \infty}(f) \lesssim \Delta_{\alpha}^{\infty, \infty}(f)
$$

Recall that

$$
\mathcal{P} f(x, t)=\int_{y \in \mathbb{R}^{n}} P_{t}(x-y) f(y) d y .
$$

Differentiating the both side, we get

$$
\nabla_{x} \mathcal{P} f(x, t)=\int_{y \in \mathbb{R}^{n}}(f(y)-\mathcal{P} f(x, t)) \nabla_{x} P_{t}(x-y) d y .
$$

By (ii) of Lemma 1.2, we have

$$
\left|\nabla_{x} \mathcal{P} f(x, t)\right| \lesssim \frac{1}{t} \int_{y \in \mathbb{R}^{n}}|f(y)-\mathcal{P} f(x, t)| P_{t}(x-y) d y .
$$

It follows that

$$
t^{1-\alpha}\left|\nabla_{x} \mathcal{P} f(x, t)\right| \lesssim t^{-\alpha} \int_{y \in \mathbb{R}^{n}}|f(y)-\mathcal{P} f(x, t)| P_{t}(x-y) d y .
$$

This implies that $L_{\alpha}^{\infty, \infty}(\nabla \mathcal{P} f) \lesssim \mathcal{G}_{\alpha}^{\infty, \infty}(f)$.

Now we state the proof for the case of $1<p, q<\infty$. By (ii) of Lemma 1.2, we have

$$
\left|\nabla_{x} \mathcal{P} f(x, t)\right| \lesssim \int_{y \in \mathbb{R}^{n}} \frac{1}{t} P_{t}(x-y)|f(y)-\mathcal{P} f(x, t)| d y .
$$

Thus it follows that

$$
\begin{aligned}
& L_{\alpha}^{p, q}(\nabla \mathcal{P} f) \\
& \leq\left(\int_{0}^{\infty}\left(t^{1-\alpha}\left(\int_{x \in \mathbb{R}^{n}}\left(\int_{y \in \mathbb{R}^{n}} \frac{1}{t} P_{t}(x-y)|f(y)-\mathcal{P} f(x, t)| d y\right)^{p} d x\right)^{1 / p}\right)^{q} \frac{d t}{t}\right)^{1 / q} \\
& =\mathcal{G}_{\alpha}^{p, q}(f) .
\end{aligned}
$$


For the converse we let

$$
\begin{aligned}
\Omega(t)= & \left(\int_{x \in \mathbb{R}^{n}}\left(\int_{y \in \mathbb{R}^{n}}|f(y)-\mathcal{P} f(x, t)| P_{t}(x-y) d y\right)^{p} d x\right)^{1 / p} \\
\lesssim & \left(\int_{x \in \mathbb{R}^{n}}\left(\int_{y \in \mathbb{R}^{n}}|f(y)-f(x)| P_{t}(x-y) d y\right)^{p} d x\right)^{1 / p} \\
& +\left(\int_{x \in \mathbb{R}^{n}}\left(\int_{y \in \mathbb{R}^{n}}|f(x)-\mathcal{P} f(x, t)| P_{t}(x-y) d y\right)^{p} d x\right)^{1 / p} \\
= & I_{1}(t)+I_{2}(t) .
\end{aligned}
$$

By Minkowski's inequality, we have

$$
\begin{aligned}
I_{2}(t) & =\left(\int_{x \in \mathbb{R}^{n}}|f(x)-\mathcal{P} f(x, t)|^{p} d x\right)^{1 / p} \\
& =\left(\int_{x \in \mathbb{R}^{n}}\left|\int_{0}^{t} \frac{\partial}{\partial s} \mathcal{P} f(x, s) d s\right|^{p} d x\right)^{1 / p} \\
& \leq \int_{0}^{t}\left\|\frac{\partial}{\partial s} \mathcal{P} f(\cdot, s)\right\|_{L^{p}} d s .
\end{aligned}
$$

By Hardy's inequality (i), we have

$$
\begin{aligned}
\left(\int_{0}^{\infty} I_{2}(t)^{q} t^{-\alpha q-1} d t\right)^{1 / q} & \lesssim\left(\int_{0}^{\infty}\left(\int_{0}^{t}\left\|\frac{\partial}{\partial s} \mathcal{P} f(\cdot, s)\right\|_{L^{p}} d s\right)^{q} t^{-\alpha q-1} d t\right)^{1 / q} \\
& \lesssim\left(\int_{0}^{\infty}\left(s\left\|\frac{\partial}{\partial s} \mathcal{P} f(\cdot, s)\right\|_{L^{p}}\right)^{q} s^{-\alpha q-1} d s\right)^{1 / q} \\
& \lesssim L_{\alpha}^{p, q}(\nabla \mathcal{P} f) .
\end{aligned}
$$

Now we estimate the first term $I_{1}(t)$. Replacing $y$ by $x+\xi$ we have

$$
\int_{y \in \mathbb{R}^{n}}|f(y)-f(x)| P_{t}(x-y) d y=\int_{\xi \in \mathbb{R}^{n}}|f(x+\xi)-f(x)| P_{t}(\xi) d \xi .
$$

By Minkowski’s inequality, we have

$$
\begin{aligned}
I_{1}(t) & =\left(\int_{x \in \mathbb{R}^{n}}\left(\int_{\xi \in \mathbb{R}^{n}}|f(x+\xi)-f(x)| P_{t}(\xi) d \xi\right)^{p} d x\right)^{1 / p} \\
& \lesssim \int_{\xi \in \mathbb{R}^{n}}\|f(\cdot+\xi)-f(\cdot)\|_{L^{p}} P_{t}(\xi) d \xi \\
& =\int_{|\xi| \leq t}\|f(\cdot+\xi)-f(\cdot)\|_{L^{p}} P_{t}(\xi) d \xi+\int_{|\xi|>t}\|f(\cdot+\xi)-f(\cdot)\|_{L^{p}} P_{t}(\xi) d \xi \\
& =I_{11}(t)+I_{12}(t) .
\end{aligned}
$$


Since $P_{t}(\xi) \lesssim 1 / t^{n}$, we have

$$
I_{11}(t)=\int_{|\xi| \leq t}\|f(\cdot+\xi)-f(\cdot)\|_{L^{p}} P_{t}(\xi) d \xi \lesssim \frac{1}{t^{n}} \int_{|\xi| \leq t}\|f(\cdot+\xi)-f(\cdot)\|_{L^{p}} d \xi .
$$

Let $\xi=r z$ where $r=|\xi|$ and $z \in \mathbb{R}^{n}$ with $|z|=1$. Then $d \xi=r^{n-1} d r d z$. Let $S^{n-1}$ be the unit sphere on $\mathbb{R}^{n}$. Let

$$
\omega(r)=\int_{S^{n-1}}\|f(\cdot+r z)-f(\cdot)\|_{L^{p}} d z .
$$

Then we have

$$
I_{11}(t) \lesssim \frac{1}{t^{n}} \int_{0}^{t} \omega(r) r^{n-1} d r
$$

Thus by Hardy's inequality (i), we have

$$
\begin{aligned}
\int_{0}^{\infty} \frac{1}{t^{1+\alpha q}} I_{11}(t)^{q} d t & \lesssim \int_{0}^{\infty} \frac{1}{t^{1+\alpha q}}\left(\frac{1}{t^{n}} \int_{0}^{t} \omega(r) r^{n-1} d r\right)^{q} d t \\
& =\int_{0}^{\infty}\left(\int_{0}^{t} \omega(r) r^{n-1} d r\right)^{q} t^{-1-\alpha q-n q} d t \\
& \lesssim \int_{0}^{\infty} \omega(r)^{q} t^{-\alpha q-1} d r \\
& =\int_{0}^{\infty}\left(\int_{S^{n-1}}\|f(\cdot+r z)-f(\cdot)\|_{L^{p}} d z\right)^{q} r^{-\alpha q-1} d r \\
& \lesssim \int_{\mathbb{R}^{n}} \frac{\|f(\cdot+\xi)-f(\cdot)\|_{L^{p}}^{q}}{|\xi|^{n+\alpha q}} d \xi
\end{aligned}
$$

Since $P_{t}(\xi) \lesssim t /|\xi|^{n+1}$, we have

$$
I_{12}(t)=\int_{|\xi|>t}\|f(\cdot+\xi)-f(\cdot)\|_{L^{p}} P_{t}(\xi) d \xi \lesssim t \int_{|\xi|>t}\|f(\cdot+\xi)-f(\cdot)\|_{L^{p}} \frac{d \xi}{|\xi|^{n+1}} .
$$

By Hardy's inequality (ii), we have

$$
\begin{aligned}
\int_{0}^{\infty} \frac{1}{t^{1+\alpha q}} I_{12}(t)^{q} d t & \lesssim \int_{0}^{\infty} \frac{1}{t^{1+\alpha q}}\left(t \int_{t}^{\infty} \omega(r) r^{-2} d t\right)^{q} d t \\
& =\int_{0}^{\infty}\left(\int_{t}^{\infty} \omega(r) r^{-2} d r\right)^{q} t^{(1-\alpha) q-1} d t \\
& \lesssim \int_{0}^{\infty}\left(\omega(r) r^{-1}\right)^{q} r^{(1-\alpha) q-1} d r \\
& \lesssim \int_{\mathbb{R}^{n}} \frac{\|f(\cdot+\xi)-f(\cdot)\|_{L^{p}}^{q}}{|\xi|^{n+\alpha q}} d \xi
\end{aligned}
$$

Therefore

$$
\mathcal{G}_{\alpha}^{p, q}(f)=\left(\int_{0}^{\infty} \frac{1}{t^{1+\alpha q}} \Omega(t)^{q} d t\right)^{1 / q} \lesssim \Delta_{\alpha}^{p, q}(f) .
$$

The other cases are similar. 
CHARACTERIZATION OF LIPSCHITZ-TYPE FUNCTIONS BY GARSIA-TYPE NORMS 79

\section{References}

[1] Sh. Axler and J. Shapiro, Putman's theorem, Alexander's spectral area estimate and VMO, Math. Ann. 271(1985), 161-183.

[2] J. B. Garnett, Bounded Analytic Functions, Academic Press, Orlando, Fla., 1981.

[3] A. Garsia, A presentation of Fefferman's theorem, Unpublished notes, 1971.

[4] E. M. Stein, Singular Integral and Differentiablity Properties of Functions, Princeton Univ. Press, Princeton, N.J., 1970.

Department of Mathematics Education, Kyungnam University, Masan, South KoREA

E-mail address: jisoobyun@gmail.com

Department of Mathematics, Pusan National University, Pusan 609-735, South KOREA

E-mail address: chohr@pusan.ac.kr

Department of Mathematics, Pusan National University, Pusan 609-735, South KOREA

E-mail address: ibk0504@pusan.ac.kr 